\title{
Designing a Provenance-Based Climate Data Analysis Application
}

\author{
Emanuele Santos ${ }^{1}$, David Koop ${ }^{1}$, Thomas Maxwell ${ }^{2}$, Charles Doutriaux ${ }^{3}$, \\ Tommy Ellqvist ${ }^{1}$, Gerald Potter ${ }^{2}$, Juliana Freire ${ }^{1}$, Dean Williams ${ }^{3}$, \\ and Cláudio T. Silva ${ }^{1}$ \\ 1 Polytechnic Institute of New York University \\ 2 NASA Goddard Space Flight Center \\ 3 Lawrence Livermore National Laboratory \\ http://uv-cdat.llnl.gov/
}

\begin{abstract}
Climate scientists have made substantial progress in understanding Earth's climate system, particularly at global and continental scales. Climate research is now focused on understanding climate changes over wider ranges of time and space scales. These efforts are generating ultra-scale data sets at very high spatial resolution. An insightful analysis in climate science depends on using software tools to discover, access, manipulate, and visualize the data sets of interest. These data exploration tasks can be complex and time-consuming, and they frequently involve many resources from both the modeling and observational climate communities. Because of the complexity of the explorations, provenance is critical, allowing scientists to ensure reproducibility, revisit existing computational pipelines, and more easily share analyses and results. In addition, as the results of this work can impact policy, having provenance available is important for decision-making. In this paper we describe, UV-CDAT, a workflow-based, provenance-enabled system that integrates climate data analysis libraries and visualization tools in an end-to-end application, making it easier for scientists to integrate and use a wide array of tools.
\end{abstract}

\section{Introduction}

This is the first paper describing capabilities of the newly developed UV-CDAT system, an advanced application that can locally and remotely access ultra-scale climate data archives, provide high-performance parallel analysis and visualization capabilities to the desktop of a climate scientist, and ultimately, apply these tools to make informed decisions on meeting the energy needs of the nation and the world in light of climate change consequences. UV-CDAT has been developed in response to the needs of scientists for access, analysis, and visualization to computer model output resulting from high-resolution, long-term, climate change projections performed as part of the U.S. Global Change Research Program. This program is funding a multi-agency effort towards the modeling and simulation of long-term climate change, and for the past several years, this effort

P. Groth and J. Frew (Eds.): IPAW 2012, LNCS 7525, pp. 214-219, 2012.

(C) Springer-Verlag Berlin Heidelberg 2012 
has been an extremely important resource for the research community. As an example of the research progress that has been enabled under this effort, the DOE BER-funded Program for Climate Model Diagnosis and Intercomparison (PCMDI) has collected and disseminated Model Intercomparison Project (MIP) simulation output from most of the world's premier climate modeling centers, including the Coupled Model Intercomparison Project, phase 3 (CMIP-3) collections which encompass over 35 terabytes (TB) of data, and more than 1 petabyte (PB) of CMIP-3 data has been distributed to over 4,300 users worldwide, resulting in over 600 peer-reviewed publications evaluating and using simulations from these state-of-the-art climate models.

Leading domain-specific tools [3, 5, 8, such as Climate Data Analysis Tools (CDAT) lack a number of desirable features to enable the analysis of this data. In particular, CDAT is ill-equipped to process very large data sets resulting from future high-resolution climate model simulations, and it lacks provenance and workflow functionality [4, 6] that are key to ensure that results are reproducible and easily accessible across the climate research community. UV-CDAT is built on top of a provenance-enabled workflow system, and all its functionality is integrated through either tightly coupled or loosely coupled software components. This model has allowed us to create a modular design that easily supports the integration of major new packages (and related functionality) in a matter of a few days versus months of efforts rewriting the guts of the system to accommodate for the new software.

To summarize, our main contribution in this paper is to describe the UVCDAT system, the first provenance-enabled end-user visualization and analysis tool. UV-CDAT presents a novel architecture that seamlessly integrates workflows, provenance, climate data analysis libraries, and visualization tools in an end-to-end application.

\section{UV-CDAT Overview}

There are quite a number of components to UV-CDAT, and it is out of the scope of this paper to provide a complete description of the system. We focus on the provenance support, and on how this was enabled in a GUI-based enduser application. Below we provide a rough overview of the system. UV-CDAT is available for downloading from http://uv-cdat.llnl.gov/. UV-CDAT is a workflow-based, provenance-enabled system that integrates climate data analysis libraries and visualization tools in an end-to-end application.

The UV-CDAT framework integrates software infrastructure through two primary means (Figure 1). Tightly coupled integration of CDAT Core, VCS and VTK/ParaView infrastructure provides high-performance parallel streaming data analysis and visualization of massive climate data sets. Loosely coupled integration provides the flexibility to use tools such as VisIt, ParaView, R, and MatLab for data analysis and visualization as well as to apply customized data analysis applications within an integrated environment without modifying the main system. VisTrails provides a package mechanism to allow developers to expose their libraries (written in any language) to the system by a thin Python 


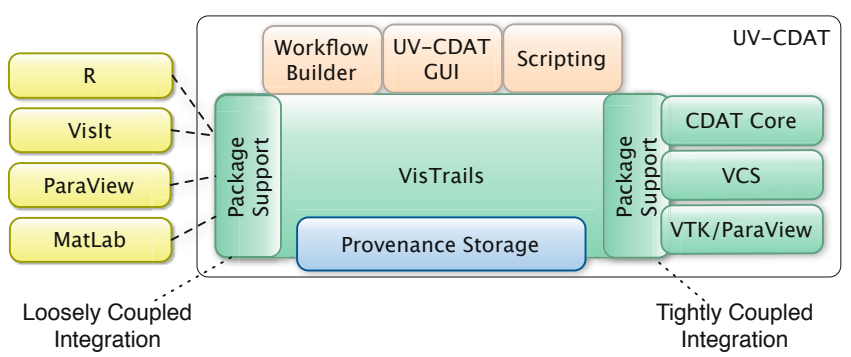

Fig. 1. UV-CDAT system architecture

interface through a set of VisTrails modules [2. In particular, the DV3D [7] system was integrated into UV-CDAT using this mechanism. DV3D provides the high-level interfaces and tools required to make the analysis and visualization power of VTK readily accessible to users without exposing visualization technical details. Within both paradigms, UV-CDAT provides data provenance capture and mechanisms to support data analysis via the VisTrails infrastructure. Users are able to interact with the system using any of the elements in the top layer: the UV-CDAT GUI, VisTrails' workflow builder or Python scripts. The UV-CDAT GUI, the main window for UV-CDAT, is shown in Figure 2 . It is based on a spreadsheet (middle), a resizable grid where each cell contains a visualization. By using intuitive drag-and-drop operations, visualizations can be created, modified, copied, rearranged, and compared. Spreadsheets maintain their provenance and can be saved and reloaded. Around the spreadsheet are the tools for building visualizations. The project view (top left) allows you to group spreadsheets into projects, and to name visualizations and spreadsheets. The plot view (bottom left) allows you to use and customize your available plot types. The variable view (top right) allows you to use and edit data variables. The bottom right contains a variable editor widget, making editing a variable similar to using a pocket calculator.

\section{UV-CDAT Provenance}

One of the key concerns in the design of UV-CDAT was integrating functionality from different sources in a way so that the provenance would be generally understandable. The two core components in accessing and visualizing information in UV-CDAT are variables and plots. A variable represents data that may be either the original data from a model or capture or the result of transforming, combining, or filtering some other data. There are many operations that allow the creation of a new variable from existing variables. A plot is a computation that generates a visualization given an input variable. In addition, it has many parameters that control the appearance of the visualization.

UV-CDAT uses the same change-based provenance to capture changes to computations as VisTrails, but users can work in an interface that is tailored to cli- 


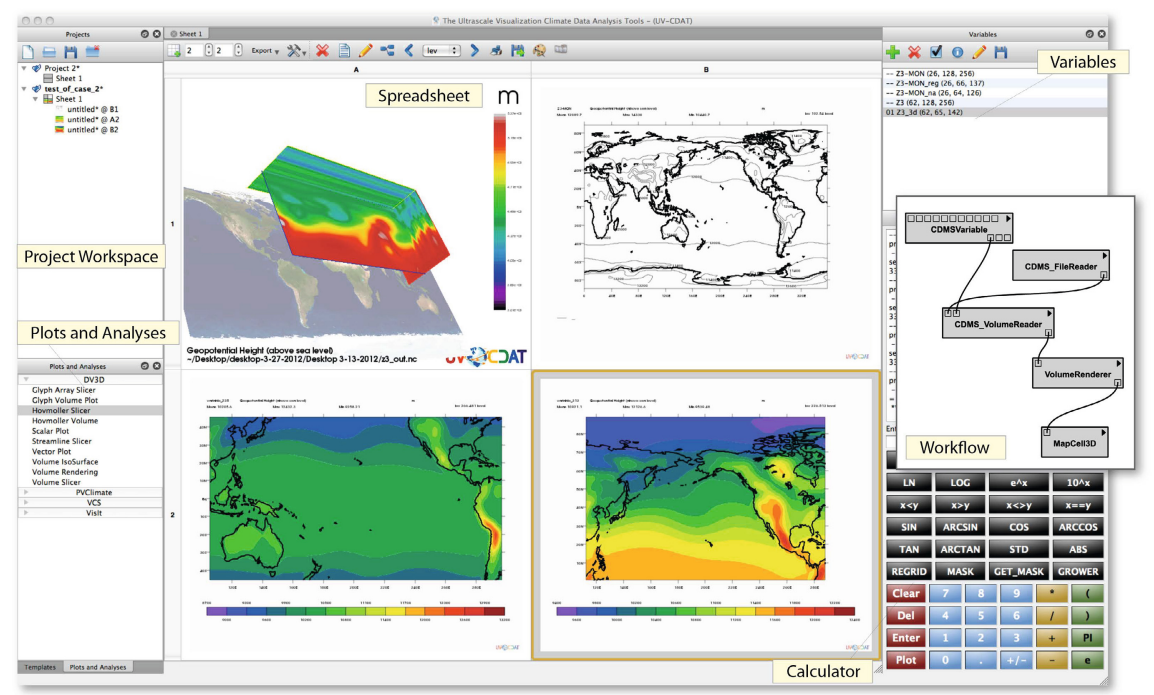

Fig. 2. UV-CDAT Main Window. Spreadsheet (middle), Project View (top left), Plot View (bottom left), Variable View (top right), and Calculator (bottom right)

mate data analysis and exploration. In order to capture provenance, UV-CDAT translates the components of the variables and plots into workflow modules which are automatically stored in a provenance format similar to VisTrails. UV-CDAT also uses the VisTrails infrastructure to capture execution provenance, capturing and storing it via the workflow execution engine. Another key requirement in the design of UV-CDAT was scripting support. We extended the provenance model to automatically generate Python scripts from the stored workflow provenance.

\section{Using UV-CDAT as an End-to-End Analysis Tool}

As a case study, we present an example of how UV-CDAT is used by a climate scientist performing data exploration and visualization. Some video tutorials can be found on http://uv-cdat.1lnl.gov/. The scientist is looking at data from paleoclimate runs on the CCSM3 [1]. The user wants to determine if the variance of the DJF (December-January-February average) $500 \mathrm{hPa}$ heights changes from two different paleoclimate simulations. This should give an indication of the changing location of storm track and could be a test of what happens to extratropical storm tracks in a warming earth. The scientist will also need to be able to do the same analysis for many different periods in the past. The list of steps performed in the analysis are the following:

1. Data discovery: The metadata for the daily model output from the model runs are examined to find the variables.

2. Select a region of interest. For example, the West Coast of the US.

3. Pick a variable and run the variance calculation on the time dimension. 
4. Save the data.

5. Plot a 3D Hovmoller diagram (latitude, longitude, time) using DV3D to see the time variation of the geopotential height.

6. Slice the data to examine the region of interest.

7. Plot 2D maps of the subregion, add overlays and manipulate plot parameters.

Figure 2 shows a few of the steps above performed in UV-CDAT. The scientist benefits from the spreadsheet by laying out different kinds of plots in the same spreadsheet. Creating 3D plots using DV3D's set of tools was a simple task. Before UV-CDAT, the scientist was required to save and manage dozens of scripts in order to know the operations and datasets used in the plots. The provenance captured in UV-CDAT is changing all that. The provenance of any plot is readily accessible at any point in time of the analysis. The scripting support was useful to generate scripts to run in batch mode for other time periods in the model run. In addition, the captured provenance allows a student not familiar with the climate model output to learn and repeat the procedure described above.

\section{Conclusion}

We have described the UV-CDAT system, what we believe is the first provenanceenabled end-user visualization and analysis tool for ultra-scale climate analysis. UV-CDAT presents a novel architecture that seamlessly integrates workflows, provenance, climate data analysis libraries, and visualization tools in an endto-end application. The system is already available to the climate community. Over the next year and a half, we will continue to refine and extend its functionality with the goal of making it the primary tool for climate scientists. Our future work plans include to further refine UV-CDAT provenance and workflow capabilities to make the integration with other packages as smoothly as possible. We plan to add a more intuitive and powerful provenance browser, and make it easier for scientists to publish their analysis, workflows, and data products on the web.

Acknowledgments. This project has been funded by the U.S. Department of Energy (DOE) Office of Biological and Environmental Research (BER). This is a large project involving many institutions, including LLNL, LBNL, Los Alamos, ORNL, Kitware, NYU-Poly, SCI-Utah, and NASA.

\section{References}

1. Community Climate System Model version 3.0 (CCSM3), http://www.cesm.ucar.edu/models/ccsm3.0/ (accessed on March 21, 2012)

2. VisTrails. In: Brown, A., Wilson, G. (eds.) The Architecture of Open Source Applications: Elegance, Evolution, and a Few Fearless Hacks, ch. 23, pp. 377-394. Lulu.com (2011), http://www.aosabook.org/

3. Climate Data Analysis Tools (CDAT), http://www2-pcmdi.llnl.gov/cdat (accessed on March 21, 2012) 
4. Davidson, S.B., Freire, J.: Provenance and Scientific Workflows: Challenges and Opportunities. In: Proceedings of SIGMOD, pp. 1345-1350 (2008)

5. Doty, B., Kinter III, J.L.: The Grid Analysis and Display System (GrADS): A practical tool for Earth science visualization. In: Eighth International Conference on Interactive Information and Procession Systems, Atlanta, GA (January 1992)

6. Freire, J., Koop, D., Silva, C.: Provenance for computational tasks: A survey. Computing in Science and Engineering 10(3), 11 (2008)

7. NASA. Dv3d, http://portal.nccs.nasa.gov/DV3D

8. Unidata. The Integrated Data Viewer (IDV), https://www. unidata.ucar.edu/software/idv/ (accessed on March 21, 2012) 